\title{
Right atrial lesions do not improve the efficacy of a complete left atrial lesion set in the surgical treatment of atrial fibrillation, but they do increase procedural morbidity
}

\author{
Lori K. Soni, MD, Sophia R. Cedola, BS, Jacob Cogan, BA, Jeffrey Jiang, BS, Jonathan Yang, MD, \\ Hiroo Takayama, MD, and Michael Argenziano, MD
}

\begin{abstract}
Objective: Some have suggested the superiority of biatrial versus left atrial lesions. We sought to analyze our experience.
\end{abstract}

\begin{abstract}
Methods: We retrospectively reviewed 305 consecutive patients from 2007 to 2011 . Rhythm success was defined as freedom from atrial fibrillation (AF) or flutter determined by 12-lead electrocardiograms at 3-month intervals. Lesions sets were pulmonary vein isolation (PVI), left-extended (PVI + mitral valve annulus [MV] lesion \pm left atrial appendage lesion [LAA]) or biatrial-extended (right atrial ablation $+\mathrm{PVI}+\mathrm{MV} \pm \mathrm{LAA}$ ).
\end{abstract}

Results: The success rates of PVI, left-extended, and biatrial-extended lesions were as follows: at 3 months, $56.7 \%, 74.7 \%$, and $79.4 \%(P=.003)$; at 6 months, $56.9 \%, 72.9 \%$, and $74.6 \%(P=.02)$; at 9 months, $54.6 \%, 72.5 \%$, and $83.3 \%(P<.001)$; and at 12 months, $52.6 \%, 76.1 \%$, and $80.0 \%(P<.001)$. Biatrial lesions had a higher rate of pacemaker placement than did left atrial lesions $(16.5 \%$ vs $7.5 \% ; P=.02)$. When we grouped patients by left lesion (PVI, PVI + MV, PVI + MV + LAA) irrespective of right atrial ablation, success was as follows: 3 months, $57.9 \%, 71.1 \%$, and $87.8 \%(P<.01)$; 6 months, $58.1 \%, 71.6 \%$, and $77.6 \%(P=.03)$; 9 months, $55.9 \%, 71.3 \%$, and $89.6 \%(P<.01)$; and 12 months, $54.1 \%, 74.7 \%$, and $83.7 \%(P<.01)$.

Conclusions: PVI is associated with lower rhythm success than an extended left atrial lesion set. The addition of a right atrial lesion to an extended left atrial lesion set does not improve efficacy, but it does increase the rate of pacemaker placement for sinus dysfunction. Adding an LAA lesion may confer additional efficacy when added to a lesion set that includes PVI + MV. (J Thorac Cardiovasc Surg 2013;145:356-63)

Atrial fibrillation (AF), the most common arrhythmia, is prevalent in $5.9 \%$ of persons older than 65 years. ${ }^{1}$ The absolute number of persons with AF will continue to rise as our population ages. In addition to the discomforting symptoms of palpitations, shortness of breath, anxiety, and reduced exercise tolerance, AF gives rise to hemodynamic and physiologic changes resulting in tachycardia-induced cardiomyopathy or thrombus formation and subsequent stroke. AF is an independent predictor of mortality. ${ }^{2}$ Furthermore, the economic burden of AF treatment in the United States is significant with an annual cost of $\$ 6.65$ billion. ${ }^{3}$

A variety of surgical approaches to AF treatment have been introduced. In an effort to decrease procedure

From Division of Cardiothoracic Surgery, Department of Surgery, Columbia University, New York, NY.

Disclosures: Authors have nothing to disclose with regard to commercial support.

Read at the 92nd Annual Meeting of The American Association for Thoracic Surgery, San Francisco, California, April 28-May 2, 2012.

Received for publication May 30, 2012; revisions received Sept 5, 2012; accepted for publication Sept 13, 2012.

Address for reprints: Lori K. Soni, MD, Division of Cardiothoracic Surgery, Department of Surgery, Columbia University, New York, NY 10032 (E-mail: 1s2932@ columbia.edu).

$0022-5223 / \$ 36.00$

Copyright (c) 2013 by The American Association for Thoracic Surgery

http://dx.doi.org/10.1016/j.jtcvs.2012.09.091 complexity, time, and risk, ${ }^{4}$ the cut-and-sew Cox maze III procedure has been replaced with modified procedures that use ablative energy sources. Some have called this variation the Cox maze IV procedure. Although the Cox maze IV procedure replicates the full biatrial lesion pattern of the cut-and-sew maze, ${ }^{5}$ a variety of less extensive variations, including procedures that include only left atrial lesions, are frequently performed.

Despite the popularity of ablative procedures limited only to the left atrium, some have suggested that left atrial lesion sets are inadequate and inferior to more complete, biatrial approaches. ${ }^{6-10}$ For this reason, we sought to analyze our experience with a variety of approaches for biatrial versus left atrial AF ablation.

\section{METHODS}

From January 2007 to November 2011, 305 consecutive patients underwent surgical ablation for AF at 1 institution. In the majority of cases, the procedure was performed in conjunction with other cardiac procedures. Concomitant procedures included mitral valve surgery $(33.3 \%$, $\mathrm{n}=100)$, aortic valve surgery $(15.1 \%, \mathrm{n}=46)$, coronary artery bypass graft $(3.9 \%, \mathrm{n}=12)$, bypass grafting plus valve $(18.0 \%, \mathrm{n}=55), 2$ or more valves $(17.7 \%, \mathrm{n}=54)$, stand-alone ablation surgery $(4.6 \%$, $\mathrm{n}=14)$, and other $(7.8 \%, \mathrm{n}=24)$. Baseline demographics are detailed in Table 1.

Surgical ablation was performed biatrially or on the left atrium only. The decision to perform biatrial versus left atrial ablation was determined 


$$
\begin{aligned}
& \text { Abbreviations and Acronyms } \\
& \begin{aligned}
\mathrm{AF} & =\text { atrial fibrillation } \\
\mathrm{BeLA} & =\text { biatrial with extended left atrial lesions } \\
\text { eLA } & =\text { extended left atrial lesions } \\
\mathrm{LAA} & =\text { left atrial appendage } \\
\mathrm{MV} & =\text { mitral valve annulus } \\
\mathrm{PVI} & =\text { pulmonary vein isolation }
\end{aligned}
\end{aligned}
$$

by each individual surgeon. All right-sided ablation lesion sets were standardized to include 3 lesions to the tricuspid valve annulus, right atrial free wall, and intercaval line (Figure 1). Left atrial ablation procedures were categorized as either pulmonary vein isolation only (PVI) or "extended left atrial lesion set," consisting of PVI isolation plus a lesion to the mitral valve annulus (MV), with or without a lesion to the left atrial appendage (LAA).

Ninety-one patients received a biatrial lesion set, and 214 patients underwent left atrial ablation only. Energy modality for ablation consisted of cryoablation $(n=145)$, radiofrequency $(n=94)$, microwave $(\mathrm{n}=65)$, and laser $(\mathrm{n}=1)$. Operative characteristics are detailed in Table 1. Our primary end point was rhythm success defined as freedom from $\mathrm{AF}$ or atrial flutter. This was determined by 12 -lead electrocardiogram at 3, 6, 9, and 12 months after surgical ablation. Follow-up was $87 \%, 87 \%$, $85 \%$, and $84 \%$ at these time points, respectively. Missing data points at various time points were left blank and not counted as procedure success or failure. There was no difference among groups as divided by lesion set with regard to completeness of follow-up. Freedom from antiarrhythmics was not incorporated in the primary end point. Secondary end points included mortality and hospital length of stay, as well as postoperative myocardial infarction, stroke, and pacemaker placement. This study was approved by our institutional review board and used informed consent.

The LAA was managed in a few ways. If the base of the appendage was noted to be broad, it was left open. Otherwise, the appendage was oversewn with a 2-layer closure. Alternatively, it was amputated with a stapler or excised and oversewn.

\section{Statistical Analysis}

Outcomes were compared using $\chi^{2}$ or Fisher's exact tests for categorical variables and the Student $t$ test for continuous variables expressed as mean \pm standard deviation. Differences in baseline demographics or operative characteristics were accounted for by stepwise backward logistic regression. Univariate variables with a significance of $P<.10$ were entered in a backward stepwise logistic regression. Stata version 11 (Stat Corporation, College Station, Tex) was used for statistical analysis.

\section{RESULTS \\ Patient Characteristics}

Our surgical cohort consisted of 305 patients, of whom $29.8 \%(\mathrm{n}=91)$ had biatrial ablation and $70.2 \%$ $(\mathrm{n}=214)$ had left atrial ablation. Baseline characteristics were similar between groups, except the left-sided ablation cohort tended to be older $(68 \pm 12$ vs $59 \pm 28 ; P<.01)$ and more frequently white $(78.0 \%$ vs $64.8 \% ; P=.02)$ and hypertensive $(73.8 \%$ vs $55.0 \% ; P<.01)$, and patients in the biatrial group had a higher incidence of prior cardiac surgery $(23.1 \%$ vs $13.1 \% ; P=.03)$. With regard to operative characteristics, biatrial ablation was more frequently performed via sternotomy as opposed to a minimally invasive approach $(87.6 \%$ vs $76.9 \% ; P=.03)$ and with an equal distribution of energy modalities, including cryoablation $(33.6 \%)$, radiofrequency $(36.9 \%)$ and microwave $(29 \%)$. The biatrial ablation group had longer cardiopulmonary bypass times ( $152 \pm 34$ minutes vs $136 \pm 54$ minutes; $P=.03$ ) and crossclamp times ( $98 \pm 41$ minutes vs $86 \pm 41$ minutes; $P=.02$ ). Patients whose concomitant procedure was mitral valve surgery were more likely to have left atrial lesions $(38 \%$ vs $21 \% ; P<.01)$.

\section{Biatrial Versus Left Atrial Lesions Sets}

First, we compared patients having biatrial lesions with those having left atrial ablations. Rhythm success was superior in the biatrial cohort at all time points $(80.3 \%$ vs $64.0 \%$ at 3 months, $P=.01 ; 75.7 \%$ vs $63.4 \%$ at 6 months, $P=.06$; $84.1 \%$ vs $61.9 \%$ at 9 months, $P<.01$; and $80.9 \%$ vs $62.4 \%$ at 12 months, $P<.01$ ).

Because a range of left atrial lesion sets was used in our patient cohort, from isolated PVI to more extensive left atrial lesion patterns, we sought to determine the contribution of this variability to rhythm success. In our biatrial cohort, $95 \%(\mathrm{n}=86)$ had extended left-sided lesions as compared with $40 \%(n=86)$ in the left-sided ablation group. In the biatrial group, 40 patients had 3 lesions $($ PVI + MV + LAA $), 46$ patients had 2 lesions $(\mathrm{PVI}+\mathrm{MV})$, and 5 patients had only PVI. In the leftsided ablation group, 22 patients had 3 lesions, 64 patients had 2 lesions, and 128 patients had only PVI (Figure 2).

Next, the cohort was divided into 3 groups: PVI only (PVI), extended left atrial lesions (eLA), and biatrial with extended left atrial lesions (BeLA). The success rates of PVI, eLA, and BeLA lesions were as follows: 3 months, $56.7 \%, 74.7 \%$, and $79.4 \%(P=.003) ; 6$ months, $56.9 \%$, $72.9 \%$, and $74.6 \%(P=.02) ; 9$ months, $54.6 \%, 72.5 \%$, and $83.3 \%(P<.001)$; and 12 months, $52.6 \%, 76.1 \%$, and $80.0 \%(P<.001)$. Through 12 months, PVI was inferior to eLA or BeLA lesions. However, there was no difference in success over this time period between patients receiving biatrial or left-sided cohorts when the left lesions were more complete (eLA) (Figure 3).

To account for differences among group characteristics, we performed a stepwise backward multivariable logistic regression. This model took into account differences in demographic characteristics including age, race, history of hypertension, and previous cardiac surgery as well as differences in operative characteristics including differences in energy modality, cardiopulmonary bypass time, and crossclamp time. As compared with a biatrial extended lesion set, PVI had inferior rhythm success at 3 months (odds ratio $[\mathrm{OR}], 0.34$; confidence interval $[\mathrm{CI}], 0.17-0.69 ; P=.003), 6$ months (OR, 0.45; CI, $0.23-0.88 ; P=.02), 9$ months (OR, 0.24; CI, 0.11-0.51; $P=.001)$, and 12 months (OR, 0.28; CI, 0.13-0.58; $P=.001)$. In our multivariable analysis, there was no 
TABLE 1. Preoperative and operative characteristics

\begin{tabular}{lccc}
\hline Preoperative characteristics & $\begin{array}{c}\text { Biatrial } \\
\text { (\% of patients) }\end{array}$ & $\begin{array}{c}\text { Left atrial } \\
\text { (\% of patients) }\end{array}$ & $\begin{array}{c}\boldsymbol{P} \\
\text { value }\end{array}$ \\
\hline No. & 91 & 214 & - \\
Age (y) & $59 \pm 28$ & $68 \pm 12$ & $<.01$ \\
Female gender & 40.7 & 45.8 & .41 \\
White race & 64.8 & 78 & .02 \\
Body mass index & $28 \pm 6$ & $27 \pm 6$ & .6 \\
Current smoker & 3.3 & 7.5 & .17 \\
Diabetes & 17.6 & 18.7 & .82 \\
Hypertension & 55 & 73.8 & $<.01$ \\
Hyperlipidemia & 27.5 & 35.6 & .23 \\
Stroke & 9.9 & 8.9 & .78 \\
Coronary artery disease & 30.8 & 42.1 & .06 \\
Congestive heart failure & 41.8 & 34.6 & .23 \\
Previous cardiac surgery & 23.1 & 13.1 & .03 \\
Depression & 5.5 & 5.1 & .89 \\
Left atrial size (cm) & $4.9 \pm 0.8$ & $5.1 \pm 0.9$ & .14 \\
AF chronicity: paroxysmal & 33.7 & 31.8 & .75 \\
Operative characteristics & & & \\
Sternotomy approach & 87.6 & 76.9 & .03 \\
Radiofrequency & 16.5 & 36.9 & $<.01$ \\
Cryoablation & 80.2 & 33.6 & $<.01$ \\
Microwave & 3.3 & 29 & $<.01$ \\
Laser & 0 & 0.47 & .51 \\
Crossclamp time (min) & $98 \pm 41$ & $86 \pm 41$ & .02 \\
CPB time (min) & $152 \pm 34$ & $136 \pm 54$ & .03 \\
\hline AF Atria fibili & & &
\end{tabular}

$A F$, Atrial fibrillation; $C P B$, cardiopulmonary bypass.

difference between eLA and BeLA groups with respect to rhythm success.

\section{Concomitant Mitral Surgery Subset}

To minimize the confounding influence of concomitant procedure heterogeneity, we repeated the analysis in the subset of our patients who had concomitant mitral valve surgery $(\mathrm{n}=100)$. In our mitral valve cohort, 19 patients had BeLA, 44 had eLA, and 37 had PVI. There were no

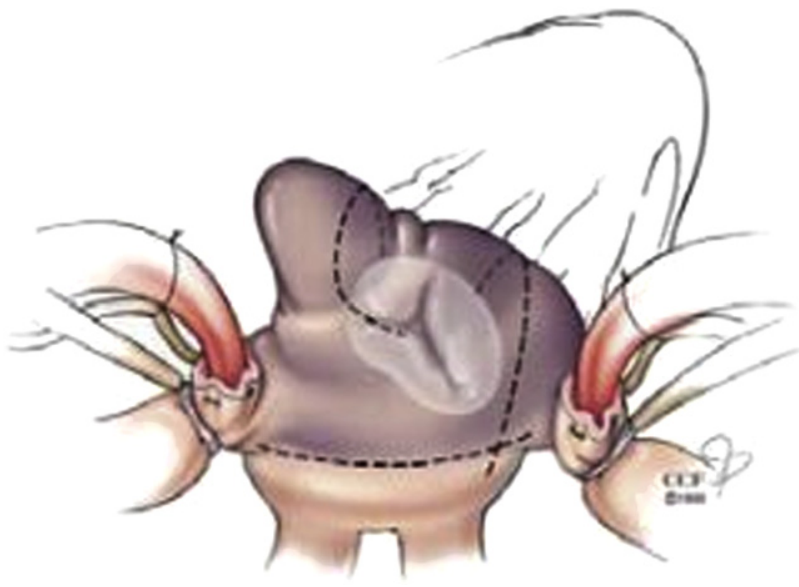

FIGURE 1. Right atrial lesion set (courtesy of Marc Gillinov, MD).

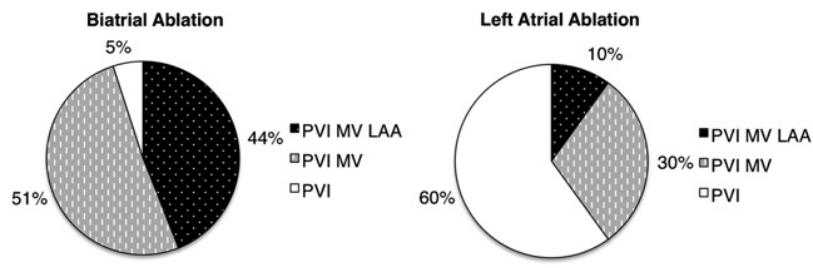

FIGURE 2. Distribution of left lesions in biatrial and left atrial lesion groups. $P V I$, Pulmonary vein isolation; $M V$, mitral valve annulus; $L A A$, left atrial appendage.

differences in baseline characteristics for this subset of patients. With respect to operative characteristics, the only significant difference was the more frequent use of cryoablation as in the BeLA group versus the eLA group $(84.2 \%$ vs $45.0 \% ; P<.01)$. Rhythm success in the PVI, eLA, and BeLA groups was as follows: at 3 months, $62.1 \%, 79.5 \%$, and $93.8 \%(P=.05)$; at 6 months, $65.5 \%$, $82.1 \%$, and $87.5 \%(P=.15)$; at 9 months, $65.5 \%, 84.2 \%$, and $93.3 \%(P=.06)$; and at 12 months, $62.1 \%, 86.5 \%$, and $86.7 \%(P=.04)$. The univariate analysis demonstrated the inferiority of PVI and no difference between eLA and BeLA lesion sets and was confirmed on multivariable logistic regression that took into account differences in energy modality.

\section{Extent of Left Atrial Lesion Set}

Next, we sought to investigate further the impact of the extent of the left atrial ablation on rhythm success. That is, did performing 3 left lesions confer greater efficacy than 2 left lesions, irrespective of whether right-sided ablation was performed? To that end, we grouped patients by left lesion set irrespective of right atrial ablation. Rhythm success for PVI, 2 left lesions, and 3 left lesions was as follows: 3 months, $57.9 \%, 71.1 \%$, and $87.8 \%(P=.001)$; 6 months, $58.1 \%, 71.6 \%$, and $77.6 \%(P=.03) ; 9$ months, $55.9 \%, 71.3 \%$, and $89.6 \%(P<.001)$; and 12 months, $54.1 \%, 74.7 \%$, and $83.7 \%(P<.001)$ (Figure 4$)$. Multivariable analysis to account for differences in baseline

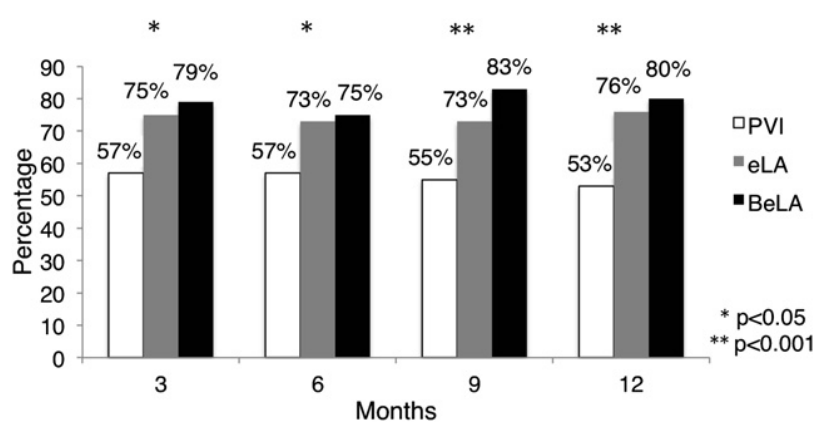

FIGURE 3. Rhythm success with pulmonary vein isolation (PVI), extended left atrial lesions $(e L A)$, and biatrial with extended left atrial lesions (BeLA). 


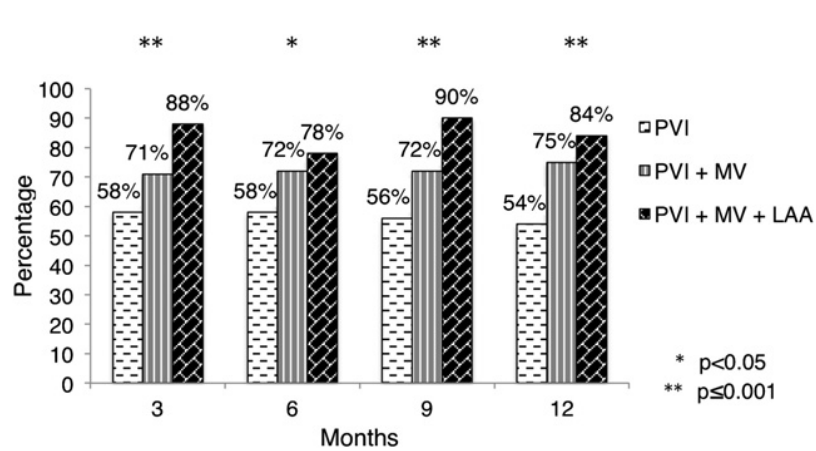

FIGURE 4. Rhythm success stratified by completeness of left atrial lesion. $P V I$, Pulmonary vein isolation; $M V$, mitral valve annulus; $L A A$, left atrial appendage.

characteristics and operative characteristics confirmed the inferiority of PVI as compared with 3 left lesions-rhythm success for PVI as compared with the 3 left lesion group was as follows: OR, 0.19; CI, 0.08-0.49 at 3 months $(P=.001)$; OR, 0.40; CI $0.18-0.87$ at 6 months $(P=.02)$; OR, 0.15; CI, $0.05-0.40$ at 9 months $(P<.001)$; and OR, 0.23; CI, 0.10-0.54 at 12 months $(P=.002)$. Multivariable analysis also suggested that 2 left lesions were inferior to 3 left lesions, at least at the 3 month (OR, 0.34; CI, 0.13-0.90; $P=.03$ ) and 9-month (OR, 0.29; CI, 0.10-0.81; $P=.02$ ) time points.

Again, we repeated this analysis in our mitral valve subset. The success of PVI, 2 left lesions, and 3 left lesions, regardless of right atrial ablation, was $62.1 \%, 79.0 \%$, and $94.1 \%$ at 3 months $(P=.04), 65.5 \%, 79.0 \%$, and $94.1 \%$ at 6 months $(P=.08), 65.5 \%, 81.1 \%$, and $100 \%$ at 9 months $(P=.02)$, and $62.1 \%, 83.3 \%$, and $93.8 \%$ at 12 months $(P=.03)$. On multivariable logistic regression, PVI was inferior to 3 left lesions at 3 months (OR, 0.10; CI, 0.01-0.88; $P=.04$ ), 6 months (OR, 0.12; CI, $0.01-1.0 ; P=.05$ ), and 12 months (OR, 0.08, CI, $0.01-0.9 ; P=.04)$, whereas there was no difference in efficacy between 2 left lesions versus 3 left lesions at 3 months (OR, 0.23; CI, 0.03-2.0; $P=.19$ ), 6 months (OR, 0.23; CI, $0.03-2.0 ; P=.19$ ), and 12 months (OR, 0.35; CI, 0.04-3.4; $P=.36)$.

\section{Morbidity}

With respect to our secondary outcomes, patients having biatrial ablation lesions had a higher rate of pacemaker placement than did those having only left atrial lesions $(16.5 \%$ vs $7.5 \% ; P=.02)$. We further investigated the

TABLE 2. Indications for Pacemaker Placement

\begin{tabular}{lrrcc}
\hline \multicolumn{1}{c}{ Indications } & eLA & BeLA & MV cohort eLA & MV cohort BeLA \\
\hline AV bock & $3.7 \%$ & $4.4 \%$ & $3.7 \%$ & $5.3 \%$ \\
Sinus Dysfunction & $3.7 \%$ & $12.1 \%$ & $4.9 \%$ & $21.1 \%$ \\
\hline
\end{tabular}

$e L A$, Extended left atrial lesions; $B e L A$, biatrial with extended left atrial lesions; $M V$, mitral valve annulus; $A V$, atrioventricular. reason for pacemaker placement to clarify whether the pacemaker placement was related to the concomitant procedure or the ablation (Table 2). Performing a 2-valve procedure at the same time as surgical ablation was a risk factor for pacemaker placement. However, there was no difference in the frequency or the type of double valve procedures between the groups to account for the different rates of pacemaker placement. In addition, other operative parameters including bypass time, energy modality, and surgeon were not predictors of postoperative pacemaker placement. In the overall patient cohort, although there was no difference in the incidence of atrioventricular block as the indication for pacemaker insertion between the biatrial versus left atrial ablation groups $(4.4 \%$ vs $3.7 \% ; P=.79)$, the incidence of sinus bradycardia or arrest was significantly higher in the biatrial group $(12.1 \%$ vs $3.7 \% ; P<.01)$. In the mitral valve subset, there was also a higher rate of pacemaker placement in the biatrial cohort as compared with the left atrial ablation cohort $(26.3 \%$ vs $8.6 \% ; P=.03)$. Again, there was no difference in the frequency of atrioventricular block as the indication for pacemaker placement in the biatrial versus the left atrial ablation groups $(5.3 \%$ vs $3.7 \%$; $P=.76)$, but there was a much higher rate of sinus dysfunction in the biatrial group $(21.1 \%$ vs $4.9 \% ; P=.02)$.

There was no difference between biatrial or left-sided ablation groups with respect to hospital length of stay $(11.2 \pm 10.9$ vs $10.5 \pm 9.1 ; P=.58)$ or 30 -day mortality $(3.8 \%$ vs $3.2 \% ; P=.80)$. There was no difference with regard to major postoperative complications including myocardial infarction $(0 \%$ vs $0.5 \% ; P=1.0)$ and stroke $(3.8 \%$ vs $3.7 \% ; P=.97)$.

\section{DISCUSSION}

In our initial analysis of our 305-patient cohort, we found a higher success rate through 12 months in those receiving biatrial versus left atrial lesion sets. This finding was consistent with those of other studies that have concluded that biatrial ablation is superior to left-sided lesions. However, these studies grouped all left lesions together ${ }^{8,11}$ and incorporated energy modalities no longer in use. When the left lesion set has been deconstructed, there have been a myriad of left lesion sets that have been compared with biatrial lesion sets. These lesion sets have included ganglionic plexi ablation, ${ }^{6}$ coronary sinus lesions, ${ }^{7}$ as well as cavotricuspid isthmus lesions. ${ }^{10}$ The results have been conflicting with reports of superior efficacy in the biatrial group $^{6,7}$ versus no difference in efficacy. ${ }^{9,12,13}$ It is difficult to compare studies given the heterogeneity of lesions performed.

Further analysis of our detailed lesion set data revealed that the overwhelming majority of the patients in our biatrial cohort had extended left lesions sets (PVI + MV \pm LAA), whereas the majority of those in the left-sided cohort had only PVI. Thus, we expanded 
our investigation to determine whether the superior efficacy observed in the biatrial cohort was attributable to the addition of a right-sided lesion, or rather to the more frequent presence of an extended left lesion set. When we broke patients down by extensiveness of the left atrial lesion, we found that the efficacy of eLA lesion set and the biatrial lesion set (BeLA) were similar and that both were superior to PVI alone. This finding conflicts with reports of satisfactory outcomes with PVI ${ }^{14-16}$ and corroborates another study that linked PVI alone to inferior outcomes. ${ }^{17}$ This may be related to the high proportion of persistent AF in our PVI cohort.

Although there were no differences in the groups with respect to important known predictors of ablation efficacy, such as left atrial size and chronicity of AF, we performed a multivariable analysis to account for other demographic and technical differences. Even after accounting for these potential confounding factors in our multivariable analysis, we found that there was no difference in success rate between an extended left atrial lesion set (eLA) and a biatrial lesion set (BeLA). These findings were also confirmed in the more homogeneous group of patients having mitral surgery as their concomitant procedure. Thus, in our patient cohort, it appears that the superior efficacy of biatrial ablation over left atrial ablation was a result of the fact that an extended left atrial lesion set (which is superior to PVI alone) was more commonly used in the biatrial procedures.

In addition, we found that 3 left lesions may confer an additional efficacy benefit as compared with 2 left lesions. Other studies have noted the MV lesion as an important addition to PVI. ${ }^{17,18}$ In some cases, the LAA is an important trigger of $\mathrm{AF}^{19}$ Our data suggests that the LAA lesion may be an important component of the left atrial lesion set in addition to the MV lesion.

We wanted to investigate the risk factors associated with postoperative pacemaker placement. We found that performing 2 valve procedures at the same time as surgical ablation was a predictor of postoperative pacemaker placement. However, there was no difference in either the frequency or type of double valve procedures in the biatrial versus the left atrial ablation cohorts. To further adjust for possible confounders, we examined pacemaker placement in our mitral valve cohort and it confirmed our finding of biatrial ablation as a predictor of postprocedure sinoatrial node dysfunction. Other operative characteristics including bypass time, energy modality, and surgeon were not predictors of postoperative pacemaker placement.

The reported rates of pacemaker placement in the literature range from $5 \%$ to $10 \%,{ }^{14,20,21}$ but few studies have reported the rate of pacemaker placement after right atrial lesions. $^{7,22} \mathrm{We}$ observed a higher rate of pacemaker placement in our biatrial cohort. There were no differences between the left-sided cohort and the biatrial cohort with respect to postprocedure atrioventricular block, and the increased pacemaker requirement in the biatrial group was related an increased incidence of sinus dysfunction. These findings were confirmed in the concomitant mitral surgery subset as well. $\mathrm{We}^{23}$ have previously published our findings noting that postoperative pacemaker placement after surgical ablation was an independent predictor of short-term mortality. Thus, this complication is not necessarily without consequence.

As this experience encompasses several years and as energy modalities in favor have changed over the years, there is a certain heterogeneity to our experience. We tried to account for these differences in energy modality in a number of ways. First, we limited our experience to the past 5 years to encompass more contemporary energy modalities and, indeed, radiofrequency and cryoablation constituted the majority of our experience. Second, we accounted for differences in energy modality in our multivariable model. Third, we performed a subanalysis on our more homogenous MV cohort.

There has been the suggestion that procedure success is dependent on the operator. We investigated this question in our experience. Surgical ablation has been performed at Columbia since the late 1990s, and our AF database has maintained records since 1999 . The operators who constituted the majority of procedures performed $(91.5 \%)$ had the same level of experience. Operator was not a predictor of procedure success.

Our study has several limitations. First, although demographic and operative data were collected prospectively in our clinical research database, all analyses were performed retrospectively. Consequently, our findings support an association between events rather than a definitive cause and effect relationship. Also, the specific procedures performed in our patients were not based on randomized assignment or even specific predetermined criteria, but rather on clinical practice norms and surgeon preference. Although we attempted to take known differences in patient characteristics and operative details into account by applying multivariable statistical techniques, our study remains vulnerable to the pitfalls of a nonrandomized, retrospective analysis, including unknown confounders and selection, procedure, and detection bias. Second, we determined rhythm success by quarterly 12-lead electrocardiographic analysis. Although this method allowed for excellent follow-up rates and represented the only practical way of getting this degree of data completeness given the demographic and geographic profile of our patients, it is subject to the risk of underdetection of arrhythmias and therefore overestimation of success rates. Third, our follow-up was only short term, although we are actively collecting long-term data. Fourth, although this study represents a large single-center cohort, the division of patients into subgroups limited our study power. Finally, our cohort includes a heterogeneous population with respect to concomitant procedures. This lends itself to 
several confounders including, potentially, differences in arrhythmia pathophysiology. A subanalysis of the mitral valve cohort attempted to correct for this, but the reduced study numbers further decreased the statistical power of this analysis.

In summary, we found that PVI is associated with lower efficacy than more extensive left atrial lesion sets and that the addition of right atrial lesions to an extended left atrial lesion set does not improve efficacy, but increases the rate of pacemaker implantation for sinus dysfunction. Given the limitations outlined above, our results are certainly not definitive. However, inasmuch as our study does describe a substantial real-world experience with a variety of lesion sets in a representative cardiac surgical population with AF, we believe that our findings are worth pursuing in a more rigorous way. We eagerly await the results of an ongoing National Institutes of Health trial studying the efficacy of left atrial and biatrial lesion sets in patients with $\mathrm{AF}$ who are undergoing mitral valve surgery.

\section{References}

1. Feinberg WM, Blackshear JL, Laupacis A, Kronmal R, Hart R. Prevalence, age distribution, and gender of patients with atrial fibrillation. Arch Intern Med. 1995; 155:469-73.

2. Kannel WB, Abbott RD, Savage DD, McNamara PM. Epidemiologic features of chronic atrial fibrillation. $N$ Engl J Med. 1982;306:1018-22.

3. Coyne KS, Paramore C, Grandy S, Mercader M, Reynolds M, Zimetbaum P. Assessing the direct costs of treating nonvalvular atrial fibrillation in the United States. Value Health. 2006;9:348-56.

4. Weimar T, Schena S, Bailey MS, Maniar HS, Schuessler RB, Cox JL, et al. The Cox-maze procedure for lone atrial fibrillation: a single-center experience over 2 decades. Circ Arrhythm Electrophysiol. 2012;5:8-14.

5. Gaynor SL, Diodato MD, Prasad SM, Ishii Y, Schuessler RB, Bailey MS, et al. A prospective, single-center clinical trial of a modified Cox maze procedure with bipolar radiofrequency ablation. J Thorac Cardiovasc Surg. 2004;128:535-42.

6. Onorati F, Mariscalco G, Rubino AS, Serraino F, Santini F, Musazzi A, et al. Impact of lesion sets on mid-term results of surgical ablation procedure for atrial fibrillation. J Am Coll Cardiol. 2011;57:931-40.

7. Kim JB, Bang JH, Jung SH, Choo SJ, Chung CH, Lee JW. Left atrial ablation versus biatrial ablation in the surgical treatment of atrial fibrillation. Ann Thorac Surg. 2011;92:1397-405.

8. Barnett SD, Niv A. Surgical ablation as treatment for the elimination of atrial fibrillation: a meta-analysis. J Thorac Cardiovasc Surg. 2006;131:1029-35.

9. Deneke T, Khargi K, Grewe PH, von Dryander S, Kuschkowitz F, Lawo T, et al. Left atrial versus bi-atrial maze operation using intraoperatively cooled-tip radiofrequency ablation in patients undergoing open-heart surgery. J Am Coll Cardiol. 2002;39:1644-50.

10. Wang J, Meng X, Li H, Cui Y, Han J, Xu C. Prospective randomized comparison of left atrial and biatrial radiofrequency ablation in the treatment of atrial fibrillation. Eur J Cardiothorac Surg. 2009;35:116-22.

11. Melo J, Santiago T, Aguiar C, Berglin E, Knaut M, Alfieri O, et al. Surgery for atrial fibrillation in patients with mitral valve disease: results at five years from the International Registry of Atrial Fibrillation Surgery. J Thorac Cardiovasc Surg. 2008;135:863-9.

12. McCarthy PM, Kruse J, Shalli S, Ilkhanoff L, Goldberger JJ, Kadish AH, et al. Where does atrial fibrillation fail? Implications for increasing effectiveness of ablation. J Thorac Cardiovasc Surg. 2010;139:860-7.

13. Albrecht A, Kalil RA, Schuch L, Abrahao R, Sant'Anna JRM, de Lima G, et al. Randomized study of surgical isolation of the pulmonary veins for correction of permanent atrial fibrillation associated with mitral valve disease. J Thorac Cardiovasc Surg. 2009;138:454-9.

14. Beyer E, Lee R, Lam B-K. Point: Minimally invasive bipolar radiofrequency ablation of lone atrial fibrillation: early multicenter results. $J$ Thorac Cardiovasc Surg. 2009; 137:521-6.
15. Edgerton JR, McClelland JH, Duke D, Gerdisch MW, Steinberg BM Bronleewe SH, et al. Minimally invasive surgical ablation of atrial fibrillation: six-month results. J Thorac Cardiovasc Surg. 2009;138:109-14.

16. Nasso G, Bonifazi R, Del Prete A, Del Prete G, Lopriore V, Bartolomucci F, et al Long-term results of ablation for isolated atrial fibrillation through a right minithoracotomy: toward a rational revision of treatment protocols. J Thorac Cardiovasc Surg. 2011;142:e41-6.

17. Gillinov AM, Bhavani S, Blackstone EH, Rajeswaran J, Svensson L, Navia JL, et al. Surgery for permanent atrial fibrillation: impact of patient factors and lesion set. Ann Thorac Surg. 2006;82:502-14.

18. Minakata K, Yunoki T, Yoshikawaa E, Katsu M, Oda T, Ujino K. Predictors of success of the modified maze procedure using radiofrequency device. Asian Cardiovasc Thorac Ann. 2011;19:33-8.

19. Biase LD, Burkhardt D, Mohanty P, Sanchez J, Mohanty S, Horton R, et al. Left atrial appendage: an underrecognized trigger site of atrial fibrillation. Circula tion. 2010;122:109-18.

20. Gammie JS, Haddad M, Milford-Beland S, Welke KF, Ferguson B, O'Brien SM et al. Atrial fibrillation correction surgery: lessons from the Society of Thoracic Surgeons National Cardiac Database. Ann Thorac Surg. 2008;85:909-15.

21. Mohr FW, Fabricius AM, Falk V, Autschbach R, Doll N, von Oppell U, et al. Curative treatment of atrial fibrillation with intraoperative radiofrequency ablation: short-term and midterm results. J Thorac Cardiovasc Surg. 2002;123:919-27.

22. Ablage A, Peterffy M, Kallner G. The biatrial cryo-maze procedure for treatment of atrial fibrillation: a single-center experience. Scand Cardiovascu Jl. 2011;45 $112-9$.

23. Worku B, Pak S-W, Cheema F, Russo M, Housman B, Van Patten D, et al. Incidence and predictors of pacemaker placement after surgical ablation for atrial fibrillation. Ann Thorac Surg. 2011;92:2085-90.

\section{Discussion}

Dr Ralph J. Damiano (St Louis, Mo). Dr Soni, I congratulate you on a beautiful presentation and for both rigorously looking at your data and acknowledging the limitations of this study. I also thank you for providing me with your slides before your talk.

In this work, Dr Argenziano and colleagues have presented a retrospective review of 305 patients undergoing primarily concomitant surgical AF ablation. They examined the impact of the lesion set performed on outcomes at 3,6, and 12 months. The principal conclusion of this study was that the efficacy of their biatrial lesion set (BeLA) was similar to that of an extended left atrial lesion set (eLA), albeit the incidence of pacemaker implantation was higher in patients who had right atrial lesions. I think this is an interesting finding and certainly suggests the need for further study. However, it is very difficult to interpret your data and almost impossible to draw any definitive conclusions.

The principal problem is that your 2 groups, patients with biatrial lesion sets and patients who just had various different left atrial lesion patterns alone, were very different in terms of their demographics, their operative characteristics, and the ablation technology that was used to create the lesions. Moreover, the 2 groups had, as you showed in one of your slides, significantly different left atrial lesion patterns. In the biatrial group (BeLA), only $5 \%$ of patients had PVI alone on the left side, whereas $60 \%$ of the patients in the left-sided alone group had just PVI as their left atrial lesion set. I would suggest that the marked differences between these 2 groups suggests there was fairly significant uncontrolled selection biases in how you decided what to do and patient and procedural differences that likely have influenced your results.

My first question for you is whether you performed any casematched comparison or tried to do a propensity analysis to control for these marked differences between the 2 groups. 
Dr Soni. That is a great question. When we first designed this study, we attempted to do a propensity analysis to account for differences among groups, which is the main limitation of a retrospective study. However, owing to the limited number of patients in the subgroupings of lesion sets, we were unable to perform a propensity analysis. In lieu of this, we adjusted for differences in patient populations using a stepwise backward multivariate logistic regression. To further adjust for confounders, we did a subanalysis of biatrial versus left lesion sets in patients who had concomitant valve surgery. In this cohort, there were no differences in baseline characteristics among the groups. Our analysis in our mitral valve cohort corroborated our findings that we presented in our larger cohort. As we expand our patient cohort over time and increase our study power, we will revisit this finding with respect to the general cohort as well as the various subgroups.

Dr Damiano. I agree. As you accumulate more patients in your series, it would be interesting to do a more detailed analysis.

The other problem, and you acknowledge this, is that the follow-up in reporting of your results does not follow the recommendations of both the 2007 and 2012 expert consensus statements on surgical and catheter ablation. Success has to be defined as freedom from both AF and atrial flutter, and I would add atrial tachycardia at 12 months. And freedom from arrhythmia has to be off antiarrhythmic drugs. Not controlling for the use of antiarrhythmic drugs, particularly in this population, would completely confuse your results. Many of these patients came in never having been on antiarrhythmic drugs because they were primarily being referred for correction of valvular or coronary disease.

If you used the correct end point, which would be freedom from both atrial tachyarrhythmias and antiarrhythmic drugs at 12 months, would you still have had the same results?

Dr Soni. With respect to antiarrhythmic drugs, that is something that we are currently evaluating. In the absence of continuous rhythm monitoring, by using spot electrocardiograms, we are going to overestimate our success. Furthermore, presenting outcomes with respect to rhythm instead of rhythm and freedom from antiarrhythmic drugs will also tend to overestimate success. However, inasmuch as the incidence of paroxysmal AF is the same in the various groups, I would not expect that we overestimated rhythm success in 1 group as compared with another. Thus, although this topic will need to be revisited, we would expect our findings to be representative.

Dr Damiano. We have found that with relatively low incidence of recurrence of AF, if you use a stricter end point, which is requiring patients to be off antiarrhythmic drugs, it is much easier to discern differences between groups.

I would ask my next quick question for you. You obviously had some patients who had prolonged monitoring. The consensus statement defined that the minimum acceptable follow-up is 24-hour Holter monitoring. Can you separate the patients who had at least 24-hour Holter monitoring and reanalyze your data? Would this change your results?

Dr Soni. That is something that we are taking a look at right now to further investigate the differences between the patients who had continuous rhythm monitoring versus those who did not.

Dr Damiano. My final quick question is that pacemaker implantation is a rough and inexact end point in that it depends on why they had the pacemaker implanted, especially when $96 \%$ of the patients had concomitant surgical procedures. Complete heart block is a complication of valve surgery; it is not a complication of a maze procedure. There is no way to get complete heart block unless you do a lesion set across the atrioventricular node, which is not described, as far as I know, in any lesion set, nor was it used in your right atrial lesion pattern. What was the indication for pacemakers in your series? How many patients had a pacemaker for complete heart block, which is a complication of the concomitant surgery, versus how many had a pacemaker put in for sinus node dysfunction, which is either a complication of the AF or potentially of the right atrial lesion set?

Dr Soni. The majority of patients had the pacemaker placed for sinus node dysfunction. This was true in our general cohort as well as the subanalysis in our mitral valve cohort. There was no difference in atrioventricular node dysfunction between biatrial or leftsided lesion set groups. This would indicate that pacemaker placement was not related to the concomitant procedure but rather the surgical ablation itself.

Dr Damiano. My point is that it is hard to blame that on the right atrial lesion set inasmuch as it cannot result in atrioventricular nodal dysfunction. It can theoretically cause sinus node dysfunction. It may be interesting to look at your data a little more carefully with this in mind.

Dr Vinay Badhwar (Pittsburgh, $\mathrm{Pa}$ ). We, like others, support your interest in homogeneity in reporting, and so I congratulate you for that.

As you know, tricuspid annular dimension may affect the decision to perform a tricuspid valve repair concomitantly at the time of a maze procedure. Have you looked at the tricuspid annular dimension in your cohort of patients who had biatrial lesion sets as well as those with left atrial only lesion sets? How many of those patients had a tricuspid valve repair and was this a factor in prompting the biatrial lesion set? If not, have you looked at late incidence of return of tricuspid regurgitation in your patient cohort in both groups and has this changed how you approach patients now, particularly in those with a tricuspid annular dimension approaching or greater than $4 \mathrm{~cm}$ ?

Dr Soni. The concomitant procedure played a role in determining the extent of the ablation. That is, when there was a reason to open the right atrium or, for that matter, the left atrium, a more limited lesion set was often performed. You raise a very interesting question with regard to the tricuspid valve. Patients who received concomitant tricuspid valve surgery actually represented a very small proportion of patients in our cohort. Furthermore, our database is a retrospective database. Although some of our data were collected prospectively with regard to operative data, we do not have data regarding the preoperative tricuspid valve annular dimension, so we have not taken a look at that. Moving forward, it would interesting evaluate that in the future.

Dr Takashi Nitta (Tokyo, Japan). I have 1 comment and a question. We did an intraoperative mapping study in the patients with AF to examine how the right atrium plays a role in sustenance of AF. The results were presented in this meeting and the paper was published in this Journal in 2005.

Most of the patients with paroxysmal AF showed a focal activation arising from the pulmonary vein and the right atrium, indicating the left atrium only lesion set would be enough to terminate AF. However, three quarters of the patients with 
chronic AF showed a reentrant activation in the right atrium, indicating the biatrial lesion set would be necessary to eliminate AF. From our data, I believe that the patients who underwent the left atrium only lesion set still have the substrate for AF in the right atrium and should be carefully followed up for the recurrence of $\mathrm{AF}$.

In our mapping study, most of the patients who showed a right atrial reentry had a dilated right atrium, only the dilated left atrium. Did you examine the effect of the size of the right atrium on the results of the left atrium only lesion set?

Dr Soni. Would you please clarify your question?

Dr Nitta. To develop the right atrial reentry, the size of the right atrium is more important than the left atrial size. Did you measure the size of the right atrium?

Dr Soni. Again, that is not something that we had measured and recorded in our database. However, we are currently taking a look at echocardiographic characteristics in both of our groups and see how that might have affected our outcomes.

Dr Ezzeldin Mostafa (Cairo, Egypt). I actually have a comment that is similar to that of my previous colleague about the morphology. I guess you have to add to the title, "in nonrheumatic patients," because rheumatic patients have a giant right atrium and a giant left atrium and sometimes rheumatic carditis to the right atrium, actually necessitating the right atrial maze. That is why we should add "in nonrheumatic patients."

My question concerns the protocol you are using. Does the protocol contain amiodarone in intraoperative and postoperative management to maintain the sinus rhythm, or do you not add amiodarone?
Dr Soni. Just to make sure I understand, you are asking about the antiarrhythmic regimen after surgery?

Dr Mostafa. Intraoperatively and postoperatively.

Dr Soni. We found that postoperative arrhythmia is not predictive of long-term rhythm success in our cohort. Postoperatively, we treat our patients with a class 1,2 , or 3 antiarrhythmic for 6 weeks. At our 6-week return visit, if they are in sinus rhythm at that point, we stop antiarrhythmic therapy. If they are not in sinus rhythm, the termination of their antiarrhythmic therapy is left up to the discretion of their cardiologist. Intraoperatively, we do not use amiodarone.

Dr Mostafa. What about the nonrheumatic pathology? In the rheumatic pathology, I do not find that an isolated left atrial lesion is complete for the revision. I guess a biatrial maze should be added.

Dr Soni. With regard to rheumatic pathology, that represents a very small percentage of patients in our cohort. We would not be able to look at that with any sort of definitive answer. When we repeated the analysis in our mitral valve cohort, we found the same results in our larger cohort.

Dr Mostafa. Thank you.

Dr Craig R. Smith (New York, NY). I have 1 quick question for my colleagues. In your conclusions you mention completeness as having some influence on the outcome. Remind us how you defined or measured completeness.

Dr Soni. The completeness was with regard to the extent of the left lesion set. A more extensive lesion set as defined by the addition of a mitral valve annulus lesion to PVI with or without an LAA lesion is predictive of success. 